\section{MRSA in Altenheimen}

Von MRSA(Methicillin-resistenter Staphylococcus aureus)-Trägern geht keine Gefahr für die Allgemeinbevölkerung aus. Somit sind auch gesunde Mitarbeiter eines Altenheimes nicht gefährdet. Routinemäßige Abstrichkontrollen bei Heimbewohnern und dem Pflegepersonal sowie ein Eingangsscreening sind nicht indiziert [1].

Die Prävalenz von MRSA-positiven Patienten bei Aufnahme in das Krankenhaus liegt bei 0,5-2 \%. Nach Köck et al. war dabei die Staphylococcus aureus-Prävalenz $32 \%$, davon waren 0,5\% MRSA-Träger [2]. MRSA-Untersuchungen beim Pflegepersonal ergaben Kolonisierungsraten für Deutschland zwischen 0,4 und $5,3 \%$.

\section{MRSA bei Alten- und Pflegeheimbewohnern}

In Deutschland liegt die durchschnittliche Besiedlung mit MRSA bei $2,4 \%$, in Nordrhein-Westfalen bei 3,1 \%. Im Großraum Frankfurt betrug die Kolonisierungsrate 9 (2007) bis 7,6\% (2012). In Wuppertal beträgt sie beispielsweise $0,79 \%[3]$.

In keinem Fall konnte eine Übertragung von einem Heimpatienten im Krankenhaus auf einen Mitpatienten oder das Pflegepersonal nachgewiesen werden. Auch gab es keine Übertragung von Keimen im Krankenhaus auf die Heimbewohner. In anderen Ländern wie den Niederlanden beträgt die Rate $0,3 \%$, in den USA 30,7 \%.

Eine Zusammenlegung von MRSA-Trägern ist möglich. Eine nähere Differenzierung der Keime sollte jedoch erfolgen.

Bei Risikogruppen von Bewohnern, die andere multiresistente Erreger (Vancomycin-resistente Enterokokken [VRE], Extended-Spectrum-Betalaktamasen [ESBL]) tragen oder infiziert sind, muss eine Zusammenlegung mit Bewohnern mit Dekubiti, Ulzera, Ekzemen, (Operations-)wunden, Kathetern, Sonden oder Tracheostoma vermieden werden.

\section{Übertragungswege}

Der wichtigste Übertragungsweg sind die Hände des Pflege- und ärztlichen Personals. Die Übertragung kann auch über Gegenstände wie beispielsweise eine Computertastatur erfolgen. Eine Erhöhung der Compliance für die Händehygiene senkt die MRSA-Übertragungsrate deutlich $(2,16$ auf 0,93 pro $10.000 \mathrm{~Pa}$ tiententage).

\section{Dekolonisierung}

Die MRSA-Dekolonisierung hat zum Ziel, eine Infektion mit dem Isolat beim Bewohner selbst und bei den anderen $\mathrm{Be}$ wohnern und beim Pflegepersonal zu verhindern. Eine Reduktion der Besiedlungsdichte durch Dekolonisierung reduziert das Übertragungsrisiko. Sie kann auch zu einer dauerhaften Sanierung führen. Die Dekolonisierung umfasst Maßnahmen im Bereich und der Umgebung von Nase, Rachen und Haut.

Die Behandlung der Nase erfolgt am besten mit Mupirocin. Nach einer Woche ist bei $90 \%$ der Patienten ein Erfolg zu verzeichnen, langfristig liegt dieser jedoch nur bei ungefähr $60 \%$. Eine Mupirocin-Behandlung über fünf Tage birgt das Risiko einer Resistenzentwicklung gegen das Antibiotikum. Im Bereich des Oropharynx ist eine dauerhafte Dekolonisierung nicht belegt. Es gibt auch keinen Beleg, dass die Dekolonisierung der Haut gelingt. Ebenfalls führen auch systemisch applizierte Antibiotika bei Dekolonisierung nicht zum Erfolg.

\section{Literatur}

1. Kommission für Krankenhaushygiene und Infektionsprävention (KRINKO) beim Robert-Koch-Institut. Empfehlungen zur Prävention und Kontrolle von Methicillin-resistenten Staphylococcus aureus-Stämmen (MRSA) in medizinischen und pflegerischen Einrichtungen. Empfehlung der Kommission für Krankenhaushygiene und Infektionsprävention (KRINKO) beim Robert-Koch-Institut. Bundesgesundheitsbl. 2014;57: 696-732.

2. Köck M et al. (2012). Vortrag auf der 52nd Interscience conference on antimicrobial agents and chemotherapy, San Francisco [Eigenes Gesprächsprotokoll].

3. Vesper F. Vorkommen von MRSA in Altenund Pflegeheimen einer bergischen Großstadt [Dissertation]. Jena: Friedrich-SchillerUniversität Jena; 2005.

Prof. Dr. med. Paul Gerhard Höher ehemaliger Direktor des Institutes für Mikrobiologie und Immunologie, Klinikum Barmen, Wuppertal gen bei männlichen wie auch weiblichen Heimbewohnern ist nicht Bestandteil des GKV-Leistungskataloges im Rahmen der Altenheimversorgung und muss daher vom Pflegepersonal des Altenheimes erbracht werden. Dies erfordert Kenntnisse, die beim Pflegepersonal nicht immer anzutreffen sind und teilweise der Schulung durch uns Urologen bedarf (Abb. 1, Abb. 2).

Eine suprapubische Fistelöffnung muss zwar offiziell nicht verbunden werden, speziell bei unruhigen Patienten ist das jedoch auch ohne lokale Entzündungszeichen sinnvoll. Die Krankenkasse übernimmt die Materialkosten jedoch nur bei Fistelkomplikationen.

Stuhl-inkontinente Patienten mit liegendem transurethralem Dauerkatheter sollten möglichst auf eine suprapubische Ableitung umgestellt werden, allerdings mit dem Risiko der traumatischen Dauerkatheterentfernung besonders bei unruhigen, dementen Bewohnern. Das Infektrisiko ist aber in jedem Falle hoch.

Nicht jede Bakteriurie muss behandelt werden, trotzdem ist es hilfreich, ein nicht zu altes Resistogramm für den Fall, dass plötzlich Symptome auftreten, zur Verfügung zu haben.

\section{Sonderfall: Heimbewohner mit multiresistenten Infekten}

Ein besonderes Problem besteht, wenn Altenheimpatienten mit multiresistenten Infekten versorgt werden müssen. Die meisten Bewohner von Altenheimen erfüllen die Kriterien eines Risikopatienten für eine MRSA(Methicillinresistente Staphylococcus aureus)-Infektion und müssten eigentlich einer MRSA-Statuserhebung zugeführt werden (stationärer Aufenthalt in den letzten sechs Monaten, chronische Pflegebedürftigkeit, Antibiotikagabe in den letzten sechs Monaten, liegende Katheter oder PEG[perkutane endoskopische Gastrostomie]-Sonde, Gangrän, chronische Wunden oder Weichteilinfektionen, Hautulzera).

Liegt eine MRSA-Trägerschaft vor, muss über eine Eradikationstherapie entschieden werden. Wie aber versorgen wir einen MRSA-positiven Patienten? Nach Möglichkeit geschieht die Versorgung vor Ort. Jedoch sind nur wenige 